\title{
OPTIMIZATION OF SODIUM DEOXYCHOLATE-BASED TRANSFERSOMES FOR PERCUTANEOUS DELIVERY OF PEPTIDES AND PROTEINS
}

\author{
ASTRIED LEONYZA, SILVIA SURINI*
}

Laboratory of Pharmaceutics and Pharmaceutical Technology Development, Faculty of Pharmacy, Universitas Indonesia, Depok, West Java, 16424, Indonesia

Email: silvia@farmasi.ui.ac.id

Received: 15 Apr 2019, Revised and Accepted: 04 Jul 2019

\section{ABSTRACT}

Objective: This study aims to formulate and characterize the transfersomes for percutaneous delivery of peptides and proteins. In particular, this study was a preliminary study for the transfersomes formulation of recombinant human epidermal growth factor (rhEGF) for topical delivery.

Methods: The transfersomes was prepared by thin film hydration method using phosphatidylcholine and sodium deoxycholate as vesicle former. In this study, transfersomes formulas were optimized, namely TF1, TF2, TF3, and TF4 with several ratios of phospholipid and surfactant which were 90:10, 85:15, 80:20, and 75:25, respectively. Afterward, the transfersomes were characterized in terms of particle size distribution, polydispersity index, zeta potential, morphology of vesicles, and deformability index.

Results: The results showed that the best formulation was TF3 with the ratio of 80:20 with a particle size of $118.6 \pm 1.33$ nm, polydispersity index of $0.102 \pm 0.011$, zeta potential of $-30.9 \pm 0,46 \mathrm{mV}$, and deformability index of $1.182 \pm 0.08$. TEM analysis also showed spherical and unilamellar vesicles of transfersomes.

Conclusion: This work demonstrated that the sodium deoxycholate-based transfersomes could be potential to be further formulated with peptide and protein for percutaneous delivery.

Keywords: Peptides, Percutaneous delivery, Proteins, Recombinant human epidermal growth factor, Sodium deoxycholate, Transfersomes

(C) 2019 The Authors. Published by Innovare Academic Sciences Pvt Ltd. This is an open access article under the CC BY license (http://creativecommons.org/licenses/by/4.0/] DOI: http://dx.doi.org/10.22159/ijap.2019v11i5.33615

Advances in biotechnology have resulted in many peptides synthesis and use of peptides and proteins in the treatment of various diseases. Currently, the most frequent route of administration for protein and peptide drugs is via parenteral because they are unstable if given orally. Transdermal delivery is an attractive option for the delivery of peptides and proteins, since it is invasive and avoids first-pass degradation in the gastrointestinal tract (GIT) and liver [1]. Besides proteins and peptides that are intended for systemic delivery, many peptides also have potential therapeutic or cosmetic value if they can be locally administered to target sites within the skin [2]. This includes epidermal growth factor (EGF) which is used for skin healing, such as chronic wounds, burns, diabetic ulcers, and also for cosmetic purposes, for example, to disguise scars, keloids, and reduce signs of skin aging [3]. In order to be effective for systemic action or as cosmeceutical products, the applied peptide must reach its target site sufficiently to generate a therapeutic response.

Meanwhile, skin administration is not an easy route because there is an effective barrier to skin penetration, called stratum corneum. It consists of a "brick and mortar" like structure with 10-15 layers of keratinocytes (bricks) in an intercellular lipid matrix (mortar) [4]. Ideal characteristics for substances intended for percutaneous delivery are a relatively low molecular weight $(<500 \mathrm{Da})$ and melting point $\left(<200^{\circ} \mathrm{C}\right)$, moderate lipophilicity (log $\mathrm{P} 1-3$ ) and aqueous solubility ( $>1 \mathrm{mg} / \mathrm{ml}$ ) and high pharmacological potency. Because of their hydrophilicity and high molecular weight, ranging from $300 \mathrm{Da}$ to greater than $1000 \mathrm{kDa}$, peptides have poor skin permeation and ineffective if administered percutaneously despite their high potency [1].

Several strategies have been developed to overcome the skin barrier and facilitate the permeation of peptides and proteins through the skin. This includes encapsulation of peptides and proteins in lipid vesicular system, such as transfersomes $[5,6]$. Transfersomes is an ultradeformable vesicle (UDV) which shows a great ability to penetrate the skin by squeeze through the pores in the stratum corneum that are less than one-tenth the diameter of the transfersomes themselves and delivering the drug to the epidermis and dermis [7]. The flexibility of transfersomes membrane is achieved by mixing suitable surface-active components (surfactants and phospholipids) in the proper ratios [8].

Sodium deoxycholate is a bile salts-derived anionic surfactant, which was employed as a potent penetration enhancer for topically administered drugs because of its membrane destabilizing activity $[9,10]$. Sodium deoxycholate has been used in a transfersomes formulation and could successfully carry insulin to penetrate across the skin [11]

In the present study, the optimization of transfersomes formulas using sodium deoxycholate as a surfactant and the characterization of the produced transfersomes were carried out. This study was a preliminary study for the formulation of recombinant human epidermal growth factor (rhEGF) in transfersomes for topical delivery.

Phospholipon 90G was a gift sample from Lipoid GmbH (Köln, Germany). Sodium deoxycholate and butylated hydroxytoluene was purchased from Sigma (Saint Louis, USA) and Sterlitamak Petrochemical Plant (Sterlitamak, Rusia) respectively. Potassium dihydrogen phosphate and sodium hydroxide were obtained from Merck (Darmstadt, Germany). All other solvents and reagents were analytical grade.

Phospholipid is the main component of vesicles forming agent, consisting of hydrophilic and hydrophobic groups, which can form a lipid bilayer arrangement in an aqueous medium [12]. Phospholipid used in this study was Phospholipon 90G, which is phosphatidylcholine from soybeans with purity of more than $94 \%$. The used phosphatidylcholine is derived from soybeans, since this type of phosphatidylcholine does not cause a smell like phosphatidylcholine derived from egg yolk. In addition to phospholipids, other components of transfersomes are surfactants/edge activators that function to increase the elasticity of vesicles, so they can deform through narrow gaps [7]. In this formulation, sodium deoxycholate (HLB 16) was chosen as an edge activator, because it has a high hydrophilic-lipophilic balance (HLB) 
value that can provide a high degree of encapsulation for hydrophilic drugs [13], such as peptides and proteins. Other surfactant with high HLB values, such as polysorbate 80 (HLB 15), was not selected as it contains ether linkages and unsaturated alkyl chains that have been shown to auto-oxidize in aqueous solution to protein-damaging peroxides and reactive aldehydes [14]. Moreover, sodium deoxycholate has been used in several studies to produce transfersomes and showed high entrapment efficiency value as well as increased flux of the penetrated drugs into skin $[15,16]$. In this study, the transfersomes vesicles were formulated with several ratios of phospholipid and surfactant as shown at table 1. Butylated hydroxytoluene (BHT) was added as an antioxidant, since transfersomes tends to experience chemical instability in the lipid phase due to oxidative degradation [12]. The $\mathrm{pH}$ of the aqueous medium was arranged with $\mathrm{pH} 7.2$ phosphate buffer solution to maintain the stability of protein and peptides.

Table 1: Formulation of the transfersomes

\begin{tabular}{|c|c|c|c|c|}
\hline \multirow[t]{2}{*}{ Material } & \multicolumn{4}{|c|}{ Concentration (\%) } \\
\hline & TF1 (90:10) & TF2 (85:15) & TF3 (80:20) & TF4 (75:25) \\
\hline Phospholipon 90G & 4.5 & 4.25 & 4 & 3.75 \\
\hline Sodium deoxycholate & 0.5 & 0.75 & 1 & 1.25 \\
\hline $\begin{array}{l}\text { Butylated hydroxytoluene } \\
\text { pH } 7.2 \text { phosphate buffer }\end{array}$ & $\begin{array}{l}0.5 \\
\text { ad } 100\end{array}$ & 0.5 & 0.5 & 0.5 \\
\hline
\end{tabular}

The transfersomes was prepared by thin layer hydration method followed by extrusion. The thin layer hydration method was applied as it is quite simple and easy to conduct on a laboratory scale [17]. Firstly, the phospholipid, surfactant, and antioxidant were dissolved in ethanol and put in a round bottom flask. The solution was subsequently evaporated using a rotary vacuum evaporator (Buchi $\mathrm{R}-100$, Switzerland) at $40{ }^{\circ} \mathrm{C}$ with the speed of $150 \mathrm{rpm}$ under vacuum condition. After the thin layer was formed, it was streamed with nitrogen gas and stored in the refrigerator overnight to allow complete evaporation of the solvent. Hydration of dry lipid film was accomplished by adding $\mathrm{pH} 7.2$ phosphate buffer solution at $37{ }^{\circ} \mathrm{C}$ with the speed of $50-250 \mathrm{rpm}$ for $45 \mathrm{~min}$. In the process of hydration, the temperature used greatly affects the stability of phospholipids and also the later active ingredient. If the temperature is too high it will disrupt the stability of the phospholipid and cause a decrease in transfersomes' encapsulation efficiency [16]. Peptides and proteins are also well known for their thermal instability. After hydration, phospholipids form a lipid bilayer vesicles of varying size and lamellarity (multilamellar vesicles/MLV) $[17,18]$. The resulting MLV transfersomes appeared as a milky white suspension.

In the end, the resulting transfersomes suspension was extruded through a polycarbonate membrane $(200 \mathrm{~nm})$. Extrusion is the most often applied method to convert MLVs to small unilamellar vesicles (SUVs) of less than $200 \mathrm{~nm}$ or large unilamellar vesicles (LUV) of
200-1000 nm [17]. The extrusion method also has the advantage of being able to produce the resulting mean vesicle size and fairly reproducible size distribution from batch-to-batch [19]. In this method, the MLV suspension is passed several times through a polycarbonate membrane with uniform pores. The average vesicle size obtained through extrusion decreases with the addition of trans-membrane pressure and the number of extrusion cycles [18]. In this study, the transfersomes suspension was extruded through $200 \mathrm{~nm}$ polycarbonate membranes (Avanti Polar Lipids Inc., USA) for 11 cycles. After extrusion, the color of the transfersomes suspension changed to be more transparent than before. It showed that increasing the surfactant concentration resulted more transparent transfersomes suspension. The appearance that became more transparent indicates that the particle size reduction has occurred. In general, the smaller the particle size, the turbidity of the colloidal system will decrease. After all the processes were complete, the transfersomes suspension was stored in a refrigerator and then characterized.

Particle size analysis and the polydispersity index of the transfersomes were measured by dynamic light scattering using a Zetasizer Nano ZS90 (Malvern, UK) instrument [20]. The particle size distribution of all transfersomes is shown at fig. 1 and the measurements of particle size as well as the polydispersity index (PDI) of the transfersomes are given in table 2.

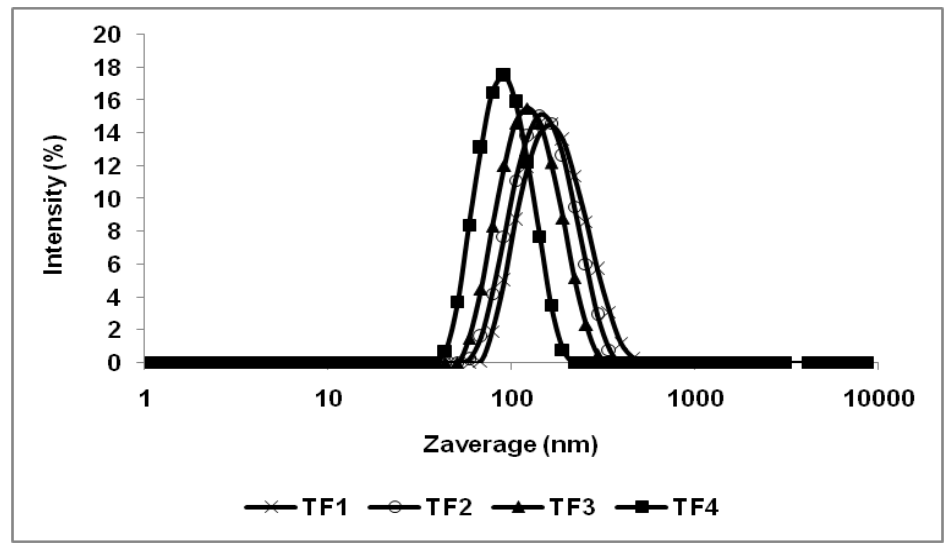

Fig. 1: Particle size distribution of the transfersomes

Table 2: Particle size distribution and polydispersity index of the thin-film

\begin{tabular}{|c|c|c|c|c|c|c|c|c|c|c|c|c|}
\hline Parameter & TF1 & & & TF2 & & & TF3 & & & TF4 & & \\
\hline $\mathrm{Z}_{\text {average }}(\mathrm{nm})$ & 156.4 & \pm & 0.85 & 138.4 & \pm & 0.55 & 118.6 & \pm & 1.33 & 86.8 & \pm & 0.14 \\
\hline $\mathrm{D}_{\mathrm{V} \text {-average }}(\mathrm{nm})$ & 200.4 & \pm & 0.76 & 167.4 & \pm & 5.86 & 132.4 & \pm & 4.24 & 81.3 & \pm & 0.57 \\
\hline $\mathrm{D}_{\mathrm{V}-10}(\mathrm{~nm})$ & 99.2 & \pm & 3.26 & 86.1 & \pm & 2.98 & 68.6 & \pm & 2.05 & 50.9 & \pm & 2.30 \\
\hline $\mathrm{D}_{\mathrm{V}-50}(\mathrm{~nm})$ & 193.3 & \pm & 0.58 & 158.0 & \pm & 5.20 & 118.7 & \pm & 2.89 & 74.9 & \pm & 1.55 \\
\hline $\mathrm{D}_{\mathrm{v}-90}(\mathrm{~nm})$ & 314.0 & \pm & 3.46 & 267.3 & \pm & 16.50 & 219.3 & \pm & 12.66 & 122.3 & \pm & 3.06 \\
\hline Polydispersity index & 0.130 & \pm & 0.011 & 0.115 & \pm & 0.008 & 0.102 & \pm & 0.011 & 0.073 & \pm & 0.018 \\
\hline
\end{tabular}

All values were represented as mean \pm SD $(n=3)$ 
All the formulation had a particle size less than $200 \mathrm{~nm}$, according to the pore size of the polycarbonate membrane used in the extrusion step. The concentration of phospholipid and surfactant used seems to be one of the most dominant parameters influencing the particle size. Surfactant concentration gives an inverse relationship with the particle size [21]. The results showed that the smallest particle size was owned by the formulation with the highest surfactant concentration (TF4<TF3<TF2<TF1). Furthermore, the reduction in polydispersity was noticed, and this might be attributed to the reduction of interfacial tension, ensuring a good emulsification process [21]. All formulations had a PDI value of less than 0.2 , indicating monodisperse particle size distribution. The extrusion method and the number of cycles that have been used are sufficient to make the transfersomes had a narrow particle size distribution.

In addition to particle size distribution and polydispersity index, the measurement of zeta potential was also conducted using a Zetasizer Nano ZS90 (Malvern, UK) instrument [20]. Zeta potential is a measure of the magnitude of the electrostatic or charges repulsion or attraction between particles in a liquid suspension. Zeta potential is a fundamental parameter to describe the stability of a dispersion system as the measurement provides detailed insight into the causes of dispersion, aggregation or flocculation. A sample with a value of zeta potential more positive than $+30 \mathrm{mV}$ or more negative than-30 $\mathrm{mV}$ can be said as stable [22]. The zeta potential values are shown in table 3 , and it revealed that the most stable formulations were TF1 and TF2, followed by TF3. The net negative charge observed was due to the lipid and surfactant composition in the formulation. Phosphatidylcholine is a zwitterionic compound with an isoelectric point between 6 and 7. During the process of formulation, $\mathrm{pH} 7.2$ phosphate buffer solution was used as the hydrating medium, where the $\mathrm{pH}$ was a little bit higher than the isoelectric point of phosphatidylcholine, causing phosphatidylcholine carried a negative charge [23, 24]. Moreover, sodium deoxycholate used is an anionic surfactant, which also contributed to the net negative charge of the formulation [13].

Table 3: Zeta potential of the transfersomes formulation

\begin{tabular}{ll}
\hline Formulation & Zeta potential (mV) \\
\hline TF1 & $-38.2 \pm 1.10$ \\
TF2 & $-38.4 \pm 1.53$ \\
TF3 & $-30.9 \pm 0.46$ \\
TF4 & $-29.4 \pm 0.66$ \\
\hline
\end{tabular}

All values were represented as mean \pm SD $(n=3)$

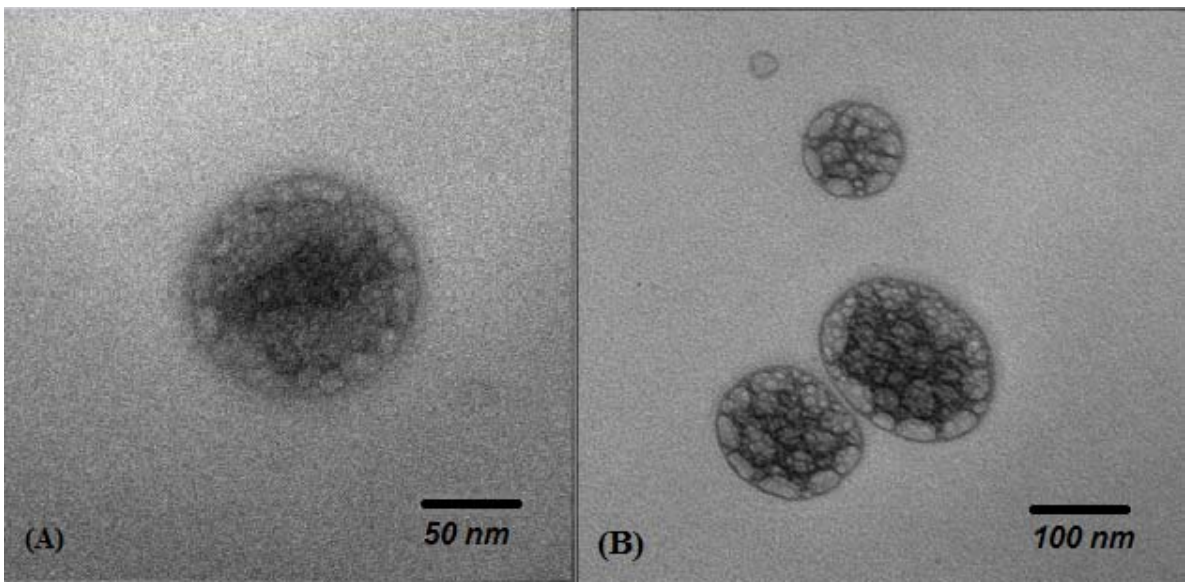

Fig. 2: TEM micrograph of the transfersomes, (A) TF1, 97,000x magnification, (B) TF3, 71,000x magnification

Morphology of vesicles was observed using a transmission electron microscope (TEM) (Microscope Tecnai 200 kV D2360 SuperTwin, Thermo Fisher Scientific, USA) with an accelerating voltage of $80 \mathrm{kV}$. An aliquot $(5 \mu \mathrm{l})$ of the transfersomes suspension was placed on a carbon-coated grid. Excess solution was carefully removed using filter paper. Transmission electron micrograph of TF1 and TF3 transfersomes are displayed at fig. 2; they show spherical and oval vesicles. When a thin film of lipid gets hydrated, it tends to form an enclosed vesicular structure with shape ranges from spherical to oval in order to attain thermodynamic stabilization by reducing the total free energy of the system. No disruption of vesicular structure confirmed vesicle integrity, even after application of various mechanical stresses such as extrusion [25]. Fig. 2 also shows that the vesicle has a unilamellar structure with a particle size of less than $200 \mathrm{~nm}$, which means the extrusion process had successfully reduced the particle size and lamellarity of transfersomes.

Deformability index is a unique parameter in transfersomes preparation that distinguishes transfersomes from other vesicular systems. Deformability index was used to examine the flexibility of transfersomes. Vesicles with better membrane deformability could penetrate the lipid membrane through the hydrophilic pathways or pores between the cells without losing their vesicle integrity. This will be possible with incorporating surfactants which destabilizes the lipid bilayer and increases its fluidity and elasticity. The transfersomes vesicles could penetrate pores that are smaller than their own diameter because of their ultra-flexible vesicle membranes [26].

Deformability index was measured by extruding $1 \mathrm{ml}$ of transfersomes suspension through a polycarbonate membrane with a pore size of $100 \mathrm{~nm}$ in a mini-extruder set (Avanti Polar Lipids Inc., USA). The extruded suspension volume in $5 \mathrm{~min}$ was recorded and the particle size was then determined with dynamic light scattering method. Deformability index can be calculated using the following equation: $\mathrm{D}=\mathrm{J}(\mathrm{rv} / \mathrm{rp})^{\wedge} 2$

where $J$ is the amount of transfersomes suspension that passed through the membrane in $5 \mathrm{~min}(\mathrm{~mL}), r v$ is the particle size of the transfersomes that passed through the membrane $(\mathrm{nm})$, and $r p$ is membrane pore size (nm) [27].

All the transfersomes suspension in the formulations were deformable, since they can pass through the membrane, and the highest deformability index owned by TF1 as shown at table 4 . 
However, these results did not show any significant value, because of the membrane pore size used does not vary much with particle sizes in the tested suspension. All the formulation experienced a reduction in particle size after extrusion in deformability test.
Nonetheless, TF3 gave the smallest reduction in particle size after extrusion. TF4 measurement was considered invalid because the pore size of the membrane used in deformability test was larger than its particle size.

Table 4: Deformability index of the transfersomes formulation

\begin{tabular}{|c|c|c|c|c|c|c|}
\hline Formulation & Volume of extruded transfersomes (ml) & \multicolumn{2}{|c|}{ Particle size after extrusion (nm) } & \multicolumn{3}{|c|}{ Deformability index } \\
\hline TF1 & 1.0 & 131.1 & 0.62 & 1.719 & \pm & 0.02 \\
\hline TF2 & 1.0 & 118.1 & 1.84 & 1.396 & \pm & 0.04 \\
\hline TF3 & 1.0 & 108.7 & 3.63 & 1.182 & \pm & 0.08 \\
\hline TF4 & 1.0 & 83.5 & 0.60 & 0.698 & \pm & 0.01 \\
\hline
\end{tabular}

All values were represented as mean $\pm S D(n=3)$

Based on the transfersomes characterizations, the selected transfersomes formulation might be determined for further formulation with an active ingredient of rhEGF or other suitable peptides and proteins. The selected formula was the transfersomes with a spherical shape and unilamellar vesicle, particle size less than $200 \mathrm{~nm}$, polydispersity index close to 0 , and zeta potential more than $\pm 30 \mathrm{mV}$. Hence, it was indicated that the best transfersomes formulation is TF3 with a ratio of phosphatidylcholine and sodium deoxycholate of 80:20, which had a spherical shape and unilamellar vesicle with a particle size of $118.6 \pm 1.33 \mathrm{~nm}$, polydispersity index of $0.102 \pm 0.011$, zeta potential of$30.9 \pm 0.46$, and the best elasticity and deformability properties. It is a potential transfersomes formula for further formulation with an active ingredient of rhEGF or other suitable peptides and proteins.

\section{ACKNOWLEDGMENT}

The authors gratefully acknowledge the PTUPT research grant support from the ministry of research, technology, and higher education, Republic of Indonesia.

\section{AUTHORS CONTRIBUTIONS}

All the authors have contributed equally

\section{CONFLICT OF INTERESTS}

The authors have no conflict of interest

\section{REFERENCES}

1. Benson HAE, Namjoshi S. Proteins and peptides: strategies for delivery to and across the skin. J Pharm Sci 2008;97:3591-610.

2. Namjoshi S, Caccetta R, Benson HAE. Skin peptides: biological activity and therapeutic opportunities. J Pharm Sci 2008;97:2524-42.

3. Caussa JE, Vila EH. Epidermal growth factor, innovation and safety. Med Clin 2015;145:305-12.

4. Bouwstra JA, Honeywell Nguyen PL, Gooris GS, Ponec M. Structure of the skin barrier and its modulation by vesicular formulations. Prog Lipid Res 2003;42:1-36.

5. Badenhorst T, Svirckis D, Wu Z. Pharmaceutical strategies for the topical dermal delivery of peptides/proteins for cosmetic and therapeutic applications. Austin J Pharmacol Ther 2014;2:1-10.

6. Chaulagain B, Jain A, Tiwari A, Verma A, Jain SK. Passive delivery of protein drugs through the transdermal route. Artif Cells Nanomed Biotechnol 2018;46 Suppl1:472-87.

7. Ascenso A, Raposo S, Catia B, Cardoso P, Mendes T, Praca FG, et al. Development, characterization, and skin delivery studies of related ultradeformable vesicles: transfersomes, ethosomes, and transethosomes. Int J Nanomed 2015;10:5837-51.

8. Prajapati ST, Patel CG, Patel CN. Transfersomes: a vesicular carrier system for transdermal drug delivery. Asian J Biochem Pharm Res 2011;1:507-24.

9. Holm R, Müllertz A, Mu H. Bile salts and their importance for drug absorption. Int J Pharm 2013;453:44-5.

10. Aburahma MH. Bile salts-containing vesicles: promising pharmaceutical carriers for oral delivery of poorly watersoluble drugs and peptide/protein-based therapeutics or vaccines. Drug Delivery 2016;23:1847-67.
11. Malakar J, Oomen S, Nayak AK. Formulation, optimization and evaluation of transferosomal gel for transdermal insulin delivery. Saudi Pharm J 2012;20:355-63.

12. Pawar A, Jadhav KR, Chaudhari LH. Transfersome: a novel technique which improves transdermal permeability. Asian J Pharm 2016;10:425-36.

13. Bnyan R, Khan I, Ehtezazi T, Saleem I, Gordon S, Francis ON, et al. Surfactant effects on lipid-based vesicles properties. J Pharm Sci 2018;107:1237-46.

14. Maggio ET. Polysorbates, peroxides, protein aggregation, and immunogenicity-a growing concern. J Excipients Food Chem 2012;3:45-53.

15. Abdallah MA. Transfersomes as a transdermal drug delivery system for enhancement the antifungal activity of nystatin. Int J Pharm Pharm Sci 2013;5:560-7.

16. Shaji J, Lal M. Praparation, optimization, and evaluation of transfersomal formulation for enhanced transdermal delivery of a COX-2 inhibitor. Int J Pharm Pharm Sci 2014;6:467-77.

17. Grimaldi N, Andrade F, Segovia N, Ferrer Tasies L, Sala S, Veciana J, et al. Lipid-based nanovesicles for nanomedicines. Chem Soc Rev 2016;45:6520-45.

18. Patil YP, Jadhav S. Novel methods for liposome preparation. Chem Phys Lipids 2014;177:8-18.

19. Lapinski MM, Castro-forero A, Greiner AJ, Ofoli RY, Blanchard GJ. Comparison of liposomes formed by sonication and extrusion: Rotational and translational diffusion of an embedded chromophore. Langmuir 2007;23:11677-83.

20. Awad RS, Abdelwahed W, Bitar Y. Evaluating the impact of preparation conditions and formulation on the accelerated stability of tretinoin loaded liposomes prepared by the heating method. Int J Pharm Pharm Sci 2015;7:171-8.

21. Singh S, Vardhan H, Kotla NG, Maddiboyina B, Sharma D, Webster TJ. The role of surfactants in the formulation of elastic liposomal gels containing a synthetic opioid analgesic. Int J Nanomed 2016;11:1475-82.

22. Malvern. Zetasizer nano series user manual. Worcestershire: Malvern Instruments Ltd; 2013.

23. Duangjit S, Opanasopit $\mathrm{P}$, Rojanarata $\mathrm{T}$, Ngawhirunpat $\mathrm{T}$. Characterization and in vitro skin permeation of meloxicamloaded liposomes versus transfersomes. J Drug Delivery 2011;2011:418316.

24. Surini S, Sarah, Djajadisastra J. Formulation and in vitro penetration study of transfersomes gel containing gotu kola leaves extract (Centella asiatica L. Urban). J Young Pharm 2018;10:27-31.

25. Yusuf M, Sharma V, Pathak K. Nanovesicles for transdermal delivery of felodipine: development, characterization, and pharmacokinetics. Int J Pharm Invest 2014;4:119-30.

26. Gonzalez Rodriguez ML, Arroyo CM, Cozar Bernal MJ, Gonzalez $\mathrm{R}$ PL, Leon JM, Calle M, et al. Deformability properties of timolol-loaded transfersomes based on the extrusion mechanism. Statistical optimization of the process. Drug Dev Ind Pharm 2016;42:1683-94.

27. Anwar E, Utami TD, Ramadon D. Transfersomal gel containing green tea (Camelia sinensis L. Kuntze) leaves extract: increasing in vitro penetration. Asian J Pharm Clin Res 2017;10:294-8. 